\title{
Intravenous Immunoglobulin Treatment in Multifocal Motor Neuropathy
}

\author{
W.-Ludo van der Pol • Elisabeth A. Cats • \\ Leonard H. van den Berg
}

Published online: 20 April 2010

(C) The Author(s) 2010. This article is published with open access at Springerlink.com

\begin{abstract}
Introduction Multifocal motor neuropathy (MMN) is characterized by asymmetric weakness of limbs and the electrophysiological finding of conduction block in motor nerves. Conduction block is the inability of nerves to propagate action potentials and is probably caused by immune-mediated dysfunction of the axon at the nodes of Ranvier or the myelin sheath. MMN immune pathogenesis has not been elucidated.

Results In approximately $50 \%$ of all patients, IgM antibodies that bind to the glycolipid GM1, which is abundantly expressed in peripheral motor nerves, can be detected. A recent study showed an association with HLA-DRB $1 * 15$, and virtually all patients respond to treatment with intravenous immunoglobulin (IVIG) in at least the early stages of the disease.

Conclusion This review aims at providing a concise overview of what is known about MMN pathogenesis, and how the beneficial effect of IVIG might be explained.
\end{abstract}

Keywords Multifocal motor neuropathy intravenous immunoglobulin · conduction block · GM1 antibodies

\section{Clinical Picture of MMN}

Asymmetric weakness of distal arm muscles without sensory deficits is a highly uncommon presentation for a

W.-L. van der Pol • E. A. Cats • L. H. van den Berg $(\bowtie)$

Rudolf Magnus Institute of Neuroscience,

Department of Neurology, University Medical Center Utrecht,

Heidelberglaan 100,

3584 CX, Utrecht, the Netherlands

e-mail: lberg@umcutrecht.nl peripheral neuropathy. Until its first detailed description in 1988 [1], multifocal motor neuropathy (MMN) was often diagnosed as amyotrophic lateral sclerosis (ALS) with a surprisingly benign disease course. ALS is a neurodegenerative disorder caused by degeneration of motor neurons in the anterior horn of the spinal cord and upper motor neurons in the central nervous system (CNS). Unlike ALS, which has a poor prognosis with a median survival of approximately 3 years, patients with MMN have a normal life expectancy, with gradual or stepwise progressive weakness of muscles in the distal arm and leg or a stable disease course after onset $[2,3]$.

Conduction block (CB) is the characteristic hallmark that distinguishes MMN from ALS and other disorders with a comparable clinical phenotype [1-3]. CB is defined as an inability of motor nerves to propagate action potentials during nerve conduction studies. Although there are many definitions of $\mathrm{CB}$, we define probable $\mathrm{CB}$ as a $30-50 \%$ reduction of the area of the compound muscle action potential (CMAP) in the arms or $>50 \%$ reduction in the legs. Definite CB is defined as a $>50 \%$ reduction of CMAP area in the arms. In the absence of $\mathrm{CB}$, a diagnosis of (possible) MMN can be made if nerve conduction studies show signs of demyelination in combination with the presence of anti-GM1 IgM antibodies in serum and abnormal magnetic resonance imaging of the brachial plexus [3]. The diagnostic criteria for MMN are summarized in Table I.

\section{Conduction Block and Nerve Pathology}

The underlying mechanisms of nerve dysfunction and, in particular, $\mathrm{CB}$ in patients with MMN are incompletely understood. $\mathrm{CB}$ could be caused by dysfunction of the axon 
Table I Proposed Diagnostic Criteria for MMN

\section{Clinical criteria}

1. Slow or stepwise progressive limb weakness

2. Asymmetrical limb weakness

3. Fewer than 7 affected limb regions (upper/lower arm, upper/lower leg on both sides, maximum 8)

4. Tendon reflexes in affected limbs are decreased or absent

5. Signs and symptoms are more pronounced in arms than in legs

6. Age 20-65 years at onset of disease

7. No objective sensory abnormalities except for vibration sense

8. No bulbar signs or symptoms

9. No upper motor neuron features

10. No other neuropathies

Laboratory criteria

1. CSF protein $<1 \mathrm{~g} / \mathrm{L}$

2. High anti-GM1 IgM titer

3. High signal intensity on T2-weighted MRI of the brachial plexus

Electrodiagnostic criteria

1. Definite motor conduction block: CMAP area reduction on proximal versus distal stimulation of at least $50 \%$ over a long segment (between erb and axilla, upper arm, lower arm, lower leg), or a CMAP amplitude reduction on proximal versus distal stimulation of at least $30 \%$ over a short distance $(2.5 \mathrm{~cm})$ detected by inching. CMAP amplitude on stimulation of the distal part of the segment with motor conduction block of at least $1 \mathrm{mV}$

2. Probable motor conduction block: CMAP amplitude reduction on proximal versus distal stimulation of at least $30 \%$ over a long segment of an arm nerve. CMAP amplitude on stimulation of the distal part of the segment with motor conduction block of at least $1 \mathrm{mV}$

3. Slowing of conduction compatible with demyelination: MCV 75\% of the lower limit of normal; DML or shortest F wave latency $130 \%$ of the upper limit of normal or absence of $\mathrm{F}$ waves all after 16-20 stimuli. CMAP amplitude on distal stimulation of at least $0.5 \mathrm{mV}$

4. Normal sensory nerve conduction in arm segments with motor conduction block. Normal SNAP amplitudes on distal stimulation

Definite MMN

1-11 on clinical criteria, 1 on laboratory criteria, 1 and 4 on electrodiagnostic criteria

Probable MMN

1-3 and 6-11 on clinical criteria, 1 on laboratory criteria, 2 and 4 on electrodiagnostic criteria

Possible MMN

1 and $7-11$ on clinical criteria, 2 or 3 on laboratory criteria, 3 and 4 on electrodiagnostic criteria

CSF cerebrospinal fluid, MRI magnetic resonance imaging, CMAP compound muscle action potential, $M C V$ motor conduction velocity, $D M L$ distal motor latency, SNAP sensory nerve action potential

Adapted from: Van Asseldonk JT, Franssen H, Van den Berg-Vos RM, Wokke JH, van den Berg LH: Multifocal motor neuropathy. Lancet Neurol. 4:309-319, 2005

or the myelin sheath, but the scarce pathological studies of motor nerves are equivocal, showing both signs of demyelination [4] and axonal degeneration [5]. Electrophysiological studies have confirmed that axonal degeneration is common in MMN $[6,7]$ and that it is the most important determinant of permanent weakness [7].

Nerve excitability studies have been used to address whether CB is caused by depolarizing or hyperpolarizing mechanisms but have yielded inconsistent results. Hyperpolarization, depolarization, and combinations have been described $[6,8]$. Depolarizing CB may precede hyperpolarizing $\mathrm{CB}$ in the course of $\mathrm{MMN}$, but these findings remain to be confirmed [8]. Importantly, axonal dysfunction in MMN may be more widespread than solely at the site of CB; this is possibly due to $\mathrm{Na}^{+}$channel dysfunction [9].
Axonal dysfunction decreased after treatment with IVIG, which suggest underlying immune-mediated mechanisms. A groundbreaking experimental study using a rabbit model for acute motor axonal neuropathy (AMAN) has provided important insight into how antibodies against GM1 disrupt the architecture of $\mathrm{Na}^{+}$channel clusters at the nodes of Ranvier [10]. By analogy, this study has offered important clues as to how immunological and electrophysiological features of MMN are linked.

\section{Anti-Ganglioside Antibodies and MMN}

The presence of serum IgM antibodies against GM1 was acknowledged in the first description of MMN [1] and has 
been since confirmed by many studies. Although the reported prevalence of IgM antibodies against GM1 in MMN patients varies widely, probably due to differences in laboratory assays [2,3], these antibodies can be detected in serum samples from approximately half of all MMN patients. GM1 is a glycolipid from the family of gangliosides, which consist of a ceramide anchor coupled to a varying number of oligosaccharide residues and sialic acids [11].

Although gangliosides are abundantly expressed in nervous tissues, their biological functions are not completely understood. From the 1980s, it is becoming increasingly clear that gangliosides are important targets for antibodies in immune-mediated neuropathies, in particular AMAN, the extensively studied pure motor axonal variant of GuillainBarré syndrome (GBS). The gangliosides GM1 and GD1a are especially abundant in motor nerves, and antibodies from all major isotypes (IgM, IgG, IgA) against these gangliosides are detected in serum from AMAN patients. These antibodies are elicited by infection with microorganisms expressing ganglioside-like mimics on their surface (a process called molecular mimicry) a few days or weeks prior to disease onset [11]. Similarly, experimental exposure to gangliosides or bacterial constituents exposing ganglioside-like structures induces inflammatory neuropathy in rabbits and humans [11]. Anti-ganglioside $\operatorname{IgG}$ antibodies were shown to induce inflammation and to disrupt nerve function by activating complement and leukocytes after binding to their targets [12].

The pathogenic role of anti-ganglioside IgM antibodies in MMN is less clear. Some have questioned the validity of analogy in MMN, pointing at the exclusive presence of the IgM isotype, the absence of these antibodies in sera from more than half of the patients with MMN, and the fact that anti-GM1 IgM antibodies are occasionally also detected in sera from patients with motor neuron disease [2]. Nevertheless, anti-GM1 IgM antibodies in sera from both GBS and MMN patients have been shown to activate complement [13]. Complement activation after binding to gangliosides is an essential step in all available models of anti-ganglioside-mediated neuropathy. The deposition of complement factors caused dysfunction of the neuromuscular junction in a mouse model [12] and induced changes in the architecture of the nodes of Ranvier and paranodal junctions, which resulted in disruption of $\mathrm{Na}^{+}$channel clusters in the rabbit model of AMAN [10]. Importantly, complement-inhibiting drugs adequately counteracted these pathogenic effects $[12,14]$. These studies suggest that antibody-complement interactions are a common final pathway in the pathogenesis of MMN and AMAN. The exclusive presence of the IgM isotype in serum from MMN patients, which generally has lower binding affinity for GM1 and reduced access through the blood-nerve barrier due to its larger size in comparison with $\mathrm{IgG}$, may explain the differences in severity of weakness between patients with MMN and AMAN. The supposed key players in MMN pathogenesis are depicted in Fig. 1.

\section{Origin of Anti-GM1 IgM Antibodies in MMN Patients}

The presence of anti-GM1 IgM antibodies in serum from almost $50 \%$ of patients with MMN seems to suggest an activation of GM1-specific B cells. Assuming that these antibodies contribute to the etiopathogenesis of MMN and are not secondary to the release of gangliosides from damaged axons, elucidation of the mechanism of GM1specific B-cell activation may provide clues for alternative treatment strategies. GM1-specific B cells may be activated by infections (molecular mimicry), intrinsic B-cell clone changes (monoclonal gammopathy), or by autoimmune mechanisms.

The almost exclusive presence of IgM and the results from serological studies, which indicate that few MMN patients had infections with the GM1-mimic expressing Campylobacter jejuni [15], do not support pathogenic similarities with AMAN. The hypothesis that MMN is a postinfectious or parainfectious disorder may be hard to investigate. The chronic disease course in MMN may cause bias in recall of individual infectious histories, and maintenance treatment with IVIG complicates the interpretation of serological studies; thus, excluding methodology that has been pivotal in the dissection of GBS pathogenesis [11]. Although monoclonal gammopathy has been observed in patients with MMN, it cannot be detected by immunofixation in the large majority of patients [2,3]. Monoclonal gammopathy of GM1-specific B cells does not, therefore, offer a satisfactory explanation for MMN in the majority of patients.

It is not clear also whether MMN is a classic autoimmune disease. Although the overrepresentation of male patients may argue against autoimmune disease, we observed a clear association of MMN with the HLADRB $1 * 15$ allele in a case-control study of 74 patients with MMN and 700 controls. Interestingly, this association is also reproducibly found in patients with multiple sclerosis, a multifocal demyelinating disease of the CNS [16]. This association may suggest pathogenic similarities with multiple sclerosis and provide a new clue for MMN pathogenesis, i.e., a contribution of antigen-presenting cells (APC). APC are pivotal in triggering adaptive immune responses by presenting autoantigens to T cells or B cells. Glycolipids are not presented by APC in an HLA-restricted fashion but by HLA-like molecules from the CD1 family. Polymorphisms of CD1 molecules were not found to be associated with MMN [17]. The association with HLA-DRB1*15 was 


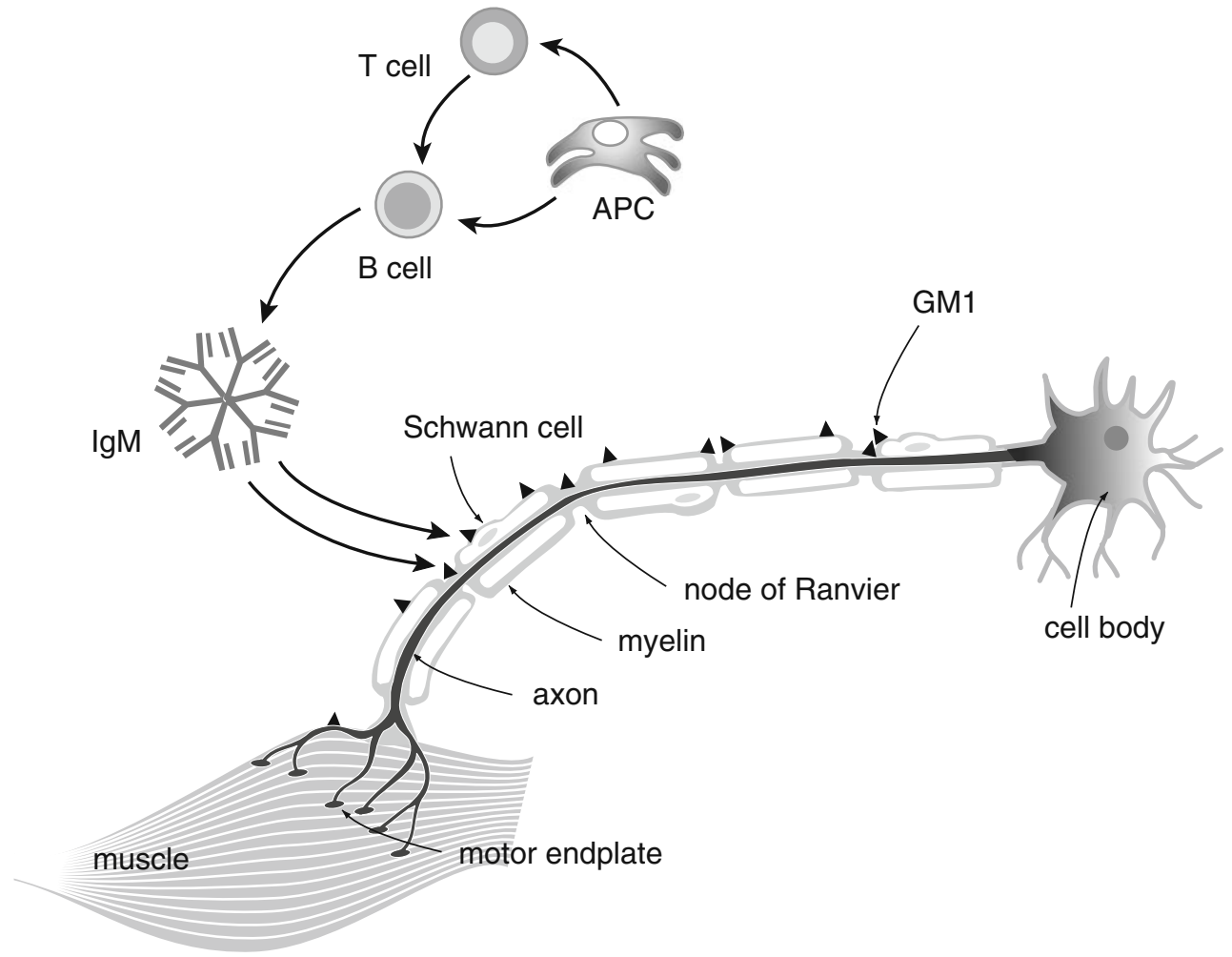

Fig. 1 Schematic depiction of assumed key players in MMN pathogenesis. Anti-GM1 IgM and possibly other antibodies are produced by $\mathrm{B}$ cells that may be specifically activated by antigen presenting cells (APC), or through "bystander" effects. There are no indications that $\mathrm{T}$ cells are involved. These IgM antibodies may bind to GM1 in nerves if the blood-nerve barrier is leaky, and may locally activate complement. The deposition of complement disrupts the

independent of the presence of anti-GM1 IgM antibodies. This may, therefore, suggest the existence of as yet unidentified autoantigens or indicate the presence of genetic risk factors that are in linkage disequilibrium with the HLA-DRB $1 * 15$ allele.

The latter possibility may be supported by the lack of evidence for T-cell involvement in MMN. Lymphocytic infiltrates were absent [4] or minute [5] in pathological studies, and serological studies have failed to show increased levels of T-cell activating interleukins (IL) such as IL-2 [18]. This is compatible with the notion that glycolipids are T-cell independent antigens and that B cells are the major culprits in the majority of immune-mediated polyneuropathies including MMN.

\section{Treatment of MMN: Present and Future}

Treatment trials have uncovered a unique pattern of responsiveness to immune modulatory treatment in MMN. Treatment with IVIG has been shown to be beneficial in randomized controlled trials. Cyclophosphamide has been architectural integrity of the nodes of Ranvier and paranodal regions, causing local disruption of $\mathrm{Na}^{+}$channel clusters. This may ultimately contribute to axonal depolarization or hyperpolarization and conduction block. IVIG might interfere with B-cell antibody production through binding to the B-cell receptor or inducing inhibitory receptors, with anti-GM1 antibodies through anti-idiotypic effects, or it might attenuate complement deposition

reported to be effective, but its toxicity precludes long-term use, which is usually necessary in patients with MMN. Plasma exchange and prednisone are well-established therapies in other immune-mediated neuropathies, but have not been shown to be effective in patients with MMN and may even aggravate symptoms $[2,3]$. This pattern supports the crucial role of B cells, antibodies, and complement in MMN pathogenesis. Cyclophosphamide is an efficient Bcell inhibiting drug. IVIG may have multiple effects on the humoral part of the immune system of MMN patients, which all contribute to its beneficial effect [19]. First, IVIG may exert anti-idiotypic effects that reduce levels of circulating anti-GM1 IgM antibodies and interfere with Bcell receptors on GM1-specific clones. Second, IVIG may induce inhibitory receptors on B cells as has been shown in patients with chronic inflammatory demyelinating polyneuropathy [20]. Finally, IVIG may interfere with antiGM1 IgM-mediated complement deposition in nerves [19]. Despite IVIG effectiveness, most patients with MMN experience mild progression of weakness during IVIG maintenance treatment of many years, which can be attributed to axonal degeneration [7]. Although IVIG 
maintenance treatment in an early stage of the disease may postpone the occurrence of permanent axonal damage [7], it is not known which dose and treatment would be optimal. In addition to studies that would optimize IVIG treatment strategies for patients with $\mathrm{MMN}$, experimental studies suggest that add-on therapy with B-cell and complementspecific agents might be successful. Anecdotal reports of the long-lasting effects of B cell-specific anti-CD20 monoclonal antibodies (rituximab) call for a randomized trial. Specific inhibitors of the complement system may represent alternative candidates [12, 14], because they might prevent permanent axonal damage. But they also may increase the risk of severe bacterial infections.

\section{Conclusion}

MMN is a rare immune-mediated neuropathy that responds to IVIG therapy. Although its pathogenesis is not completely understood, the available data strongly suggest a role for B cells, IgM antibodies, and complement. Further dissection of its etiology may help to select effective treatment modalities. Future treatment strategies should aim ultimately at preventing permanent axonal damage without increasing the risk of serious side effects.

Acknowledgments Elisabeth A. Cats is supported by a grant from the Prinses Beatrix Fonds.

Open Access This article is distributed under the terms of the Creative Commons Attribution Noncommercial License which permits any noncommercial use, distribution, and reproduction in any medium, provided the original author(s) and source are credited.

\section{References}

1. Pestronk A, Cornblath DR, Ilyas AA, Baba H, Quarles RH, Griffin JW, et al. A treatable multifocal motor neuropathy with antibodies to GM1 ganglioside. Ann Neurol. 1988;24:73-8.

2. Nobile-Orazio E. Multifocal motor neuropathy. J Neuroimmunol. 2001;115:4-18.

3. Van Asseldonk JT, Franssen H, Van den Berg-Vos RM, Wokke $\mathrm{JH}$, Van den Berg LH. Multifocal motor neuropathy. Lancet Neurol. 2005;4:309-19.

4. Corbo M, Abouzahr MK, Latov N, Iannaccone S, Quattrini A, Nemni R, et al. Motor nerve biopsy studies in motor neuropathy and motor neuron disease. Muscle Nerve. 1997;20:15-21.
5. Taylor BV, Dyck PJ, Engelstad J, Gruener G, Grant I, Dyck PJ. Multifocal motor neuropathy: pathologic alterations at the site of conduction block. J Neuropathol Exp Neurol. 2004;63:129-37.

6. Kiernan MC, Guglielmi JM, Kaji R, Murray NM, Bostock H. Evidence for axonal membrane hyperpolarization in multifocal motor neuropathy with conduction block. Brain. 2002;125: 664-75.

7. Van Asseldonk JT, Van den Berg LH, Kalmijn S, Van den BergVos RM, Polman CH, Wokke JH, et al. Axon loss is an important determinant of weakness in multifocal motor neuropathy. J Neurol Neurosurg Psychiatry. 2006;77:743-7.

8. Priori A, Bossi B, Ardolino G, Bertolasi L, Carpo M, NobileOrazio E, et al. Pathophysiological heterogeneity of conduction blocks in multifocal motor neuropathy. Brain. 2005;128:1642-8.

9. Priori A, Cinnante C, Pesenti A, Carpo M, Cappellari A, NobileOrazio E, et al. Distinctive abnormalities of motor axonal strength-duration properties in multifocal motor neuropathy and in motor neurone disease. Brain. 2002;125:2481-90.

10. Susuki K, Rasband MN, Tohyama K, Koibuchi K, Okamoto S, Funakoshi K, et al. Anti-GM1 antibodies cause complementmediated disruption of sodium channel clusters in peripheral motor nerve fibers. J Neurosci. 2007;27:3956-67.

11. Willison HJ, Yuki N. Peripheral neuropathies and anti-glycolipid antibodies. Brain. 2002;125:2591-625.

12. Halstead SK, Zitman FM, Humphreys PD, Greenshields K, Verschuuren JJ, Jacobs BC, et al. Eculizumab prevents antiganglioside antibody-mediated neuropathy in a murine model. Brain. 2008;131:1197-208.

13. Uetz-von Allmen E, Sturzenegger M, Rieben R, Rihs F, Frauenfelder A, Nydegger UE. Antiganglioside GM1 antibodies and their complement activating capacity in central and peripheral nervous system disorders and in controls. Eur Neurol. 1998;39:103-10.

14. Phongsisay V, Susuki K, Matsuno K, Yamahashi T, Okamoto S, Funakoshi K, et al. Complement inhibitor prevents disruption of sodium channel clusters in a rabbit model of Guillain-Barre syndrome. J Neuroimmunol. 2008;205:101-4.

15. Terenghi F, Allaria S, Scarlato G, Nobile-Orazio E. Multifocal motor neuropathy and Campylobacter jejuni reactivity. Neurology. 2002;59:282-4.

16. Sutedja NA, Otten HG, Cats EA, Piepers S, Veldink JH, van der Pol WL, et al. Increased frequency of HLA-DRB $1 * 15$ in patients with multifocal motor neuropathy. Neurology. 2010;74:828-32.

17. De Angelis MV, Notturno F, Caporale CM, Pace M, Uncini A. Polymorphisms of CD1 genes in chronic dysimmune neuropathies. J Neuroimmunol. 2007;186:161-3.

18. Terenghi F, Allaria S, Nobile-Orazio E. Circulating levels of cytokines and their modulation by intravenous immunoglobulin in multifocal motor neuropathy. J Peripher Nerv Syst. 2006;11:67-71.

19. Dalakas MC. Mechanisms of action of IVIg and therapeutic considerations in the treatment of acute and chronic demyelinating neuropathies. Neurology. 2002;59:S13-21.

20. Tackenberg B, Jelcic I, Baerenwaldt A, Oertel WH, Sommer N, Nimmerjahn F, et al. Impaired inhibitory Fcgamma receptor IIB expression on B cells in chronic inflammatory demyelinating polyneuropathy. Proc Natl Acad Sci USA. 2009;106:4788-92. 